\section{Contrast-enhanced ultrasound imaging features and clinical characteristics of combined hepatocellular cholangiocarcinoma: comparison with hepatocellular carcinoma and cholangiocarcinoma}

\author{
Hai-Chun Zhang ${ }^{1^{*}}$, Ting Zhu ${ }^{2^{*}}$, Rong-Fei $\mathrm{Hu}^{2}$, Long $\mathrm{Wu}^{2}$ \\ 'Department of Ultrasound, Shanghai Deji Hospital/Shanghai Neuromedical Center, Qingdao \\ University, Shanghai; ${ }^{2}$ Department of Ultrasound, Huashan North Hospital Baoshan Branch \\ Affiliated with Fudan University, Shanghai, China
}

Purpose: The purpose of this study was to retrospectively compare the clinical characteristics and imaging features on (CEUS) of combined hepatocellular cholangiocarcinoma (CHC) with those of hepatocellular carcinoma (HCC) and cholangiocarcinoma (CC).

Methods: The clinical information and CEUS features of 45 patients with CHC from 2015 to 2019 and 1-to-1-matched control subjects with HCC and CC (45 each) were compared.

Results: Simultaneous elevation of a-fetoprotein (AFP) and cancer antigen (CA) 19-9 was more common in CHC than in HCC and CC. In the arterial phase, hyperenhancement (homogeneous and heterogeneous) was more common in $\mathrm{CHC}(73.3 \%)$ and $\mathrm{HCC}(100 \%)$, while peripheral rimlike enhancement was more common in CC (55.6\%). In the portal phase, marked washout was significantly more frequent in $\mathrm{CHC}$ and $\mathrm{CC}$ than in $\mathrm{HCC}(42.2 \%$ and $53.3 \%$ vs. $6.7 \%)$. In the delayed phase, marked washout was more common in $\mathrm{CHC}(82.2 \%)$ and $\mathrm{CC}(93.3 \%)$ than in HCC (40.0\%). The washout time (WT) was much shorter in CHC and CC than in HCC (33.8 13.1 seconds and $30.1 \pm 11.6$ seconds vs. $58.4 \pm 23.5$ seconds). Using the combination of simultaneous elevation of AFP and CA 19-9 with marked washout in the delayed phase and a WT $<38$ seconds or arterial hyperenhancement to differentiate CHC from HCC or CC, the accuracy, sensitivity, and specificity were $74.4 \%, 93.3 \%$, and $55.6 \%$ and $71.1 \%, 80.0 \%$, and $62.2 \%$, respectively.

Conclusion: Although some CEUS imaging features of CHC, HCC, and CC overlap, the combination of tumor markers and CEUS features can be helpful in differentiating CHC from HCC and CC.

Keywords: Combined hepatocellular cholangiocarcinoma; Hepatocellular carcinoma; Cholangiocarcinoma; Contrast-enhanced ultrasound

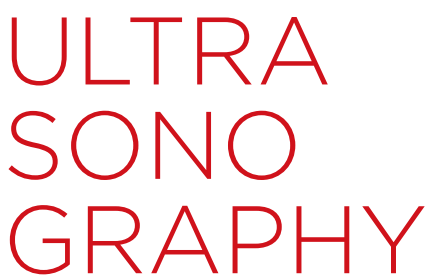

ORIGINAL ARTICLE

https://doi.org/10.14366/usg. 19093 pISSN: 2288-5919 • elSSN: 2288-5943 Ultrasonography 2020;39:356-366

Received: December 20, 2019

Revised: March 12, 2020

Accepted: March 13, 2020

* These authors contributed equally to this work.

Correspondence to:

Hai-Chun Zhang, MD, Department of Ultrasound, Shanghai Deji Hospital/ Shanghai Neuromedical Center, Qingdao University, No. 378 Gulang Road, Shanghai 200331, China

Tel. +86-130-5223-4091

Fax. +86-21-6587-8392

E-mail: 33818460@qq.com

This is an Open Access article distributed under the terms of the Creative Commons Attribution NonCommercial License (http://creativecommons.org/ licenses/by-nc/4.0/) which permits unrestricted noncommercial use, distribution, and reproduction in any medium, provided the original work is properly cited.

Copyright (C) 2020 Korean Society of Ultrasound in Medicine (KSUM)

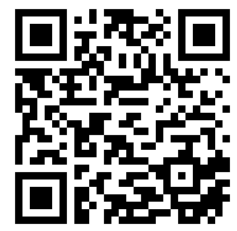

How to cite this article:

Zhang HC, Zhu T, Hu RF, Wu L. Contrastenhanced ultrasound imaging features and clinical characteristics of combined hepatocellular cholangiocarcinoma: comparison with hepatocellular carcinoma and cholangiocarcinoma. Ultrasonography. 2020 0ct;39(4):356-366. 


\section{Introduction}

Hepatocellular carcinoma (HCC) and cholangiocarcinoma (CC) account for the vast majority of primary liver malignancies, while combined hepatocellular cholangiocarcinoma (CHC)-also referred to as a "biphenotypic" tumor-comprises a distinct minority, accounting for $0.4 \%-14.5 \%$ [1]. Although CHC has been categorized into three distinct types based on the relative separation of the different CC and HCC components, only those consisting of intermixed components and transitional cell types appear to represent true combination tumors, whereas other types may exist along the spectrum of collision tumors [2]. The prognosis of CHC appears to be worse than that of $\mathrm{HCC}$ and similar to that of $\mathrm{CC}$ due to the high frequency of vascular invasion and lymph node metastasis [2-4]. At present, according to guidelines, surgical resection, transplantation, and percutaneous ablation constitute the treatment strategy for HCC [5], while for CC, resection has been regarded as the first-line approach [6]. However, the most appropriate treatment for CHC remains unclear, even though radical liver resection likely yields the greatest survival benefit in limited-stage patients [7]. Therefore, the conundrum of the preoperative diagnosis is a potential factor shaping treatment selection.

A few studies have evaluated the imaging characteristics of $\mathrm{CHC}$ based on contrast-enhanced computed tomography (CECT) $[8,9]$ and magnetic resonance imaging (MRI) $[8,10,11]$. Theoretically, because CHC comprises HCC and CC components, the imaging features of both HCC and CC would be visualized, with either an HCC-like or CC-like appearance predominating [8]. Contrastenhanced ultrasonography (CEUS) has been found to be clinically valuable in the diagnosis of focal liver lesions for years, as it can non-invasively reflect the blood perfusion of tumor tissue in real time [12]. However, only sporadic reports have investigated the CEUS features of CHC [13-16]. Furthermore, because of its rarity, limited studies have assessed the diagnostic performance of CEUS in the differential diagnosis of CHC from HCC and CC.

Regarding the clinical features, cancer antigen (CA) 19-9 and a -fetoprotein (AFP), which are useful adjuncts to imaging in patients with $C C$ and $H C C$, respectively, are the main tumor markers of interest. Although prior studies have suggested that once both AFP and CA 19-9 are simultaneously elevated or are elevated in discordance with the imaging features (mainly CECT or MRI), a diagnosis of $\mathrm{CHC}$ should be considered $[8,17]$. However, the diagnostic performance of this widely accepted algorithm for the differential diagnosis of CHC and HCC or CC has not yet been investigated.

Therefore, the purpose of this study was to evaluate the diagnostic performance of CEUS and clinical features in distinguishing $\mathrm{CHC}$ from $\mathrm{HCC}$ and $\mathrm{CC}$, and to identify preoperative clues that may indicate the diagnosis and better guide clinical management decisions.

\section{Materials and Methods}

\section{Patients}

Institutional review board approval with a consent waiver was obtained for this retrospective study. Our institutional pathology database was searched for consecutive CHC tumors between January 2015 and June 2019, and these results were crossreferenced with the radiology database, excluding any patients without preoperative CEUS. The pathology and radiology databases were also searched for HCC and CC cases over the same period. Due to the relative rarity of $\mathrm{CHC}$, the number of $\mathrm{HCC}$ and $\mathrm{CC}$ cases far exceeded that of $\mathrm{CHC}$ cases; therefore, a random number generator software tool was used to randomly choose HCC and CC cases according to a 1:1 proportion. As a result, 135 patients-including 45 with $\mathrm{CHC}, 45$ with $\mathrm{HCC}$, and 45 with $\mathrm{CC}$-were included in this retrospective study. All patients underwent hepatectomy and the diagnosis was confirmed through a postoperative pathology report.

\section{CEUS Examinations}

The CEUS examinations were performed by an experienced sonologist (R.F.H.) with more than 20 years of liver ultrasonography experience using two scanners (C1-5, 1-5 MHz, Logiq E9, GE Healthcare, Chicago, IL, USA; C5-1, 1-5 MHz, IU22, Philips Medical Systems, Foster City, CA, USA). On grayscale ultrasonography, the tumor number, location, and size were recorded. Then, CEUS was performed with a low mechanical index of $<0.1$, and $2.4 \mathrm{~mL}$ of contrast agent (SonoVue, Bracco, Switzerland) was antecubitally injected as a bolus followed by a $5-\mathrm{mL}$ saline flush. The timer was started at the contrast agent injection ( 0 second), and the lesion was scanned continuously for up to 3 minutes. As a routine examination procedure, the technical settings were fixed for CEUS: dynamic range, $65-70 \mathrm{~dB}$; frame rate, $12-15 \mathrm{fps}$; gain, $75 \%$; and one focus below the lesion. The entire vascular phase was recorded on a hard drive for further analysis.

In this study, a per-patient analysis was performed. In patients with more than one hepatic lesion, only the largest and bestvisualized lesion was targeted because CEUS could not be used to scan multiple nodules simultaneously after a single injection of contrast agent.

\section{CEUS Interpretation}

All the CEUS videos were reviewed and evaluated by two experienced sonologists (T.Z. and L.W.) in consensus. The entire 
vascular phase consisted of three phases: arterial $(0-30$ seconds after the injection), portal (31-120 seconds after the injection), and delayed phase (>120 seconds after the injection) [18].

In the arterial phase, the enhancement pattern was defined by a comparison of enhancement behavior between the tumor and liver parenchyma, and was classified as follows (Figs. 1-5): (1) homogeneous hyperenhancement: entirely hyperenhanced without any defects compared with the liver parenchyma; (2) heterogeneous hyperenhancement: mixed hyperenhancement in both the peripheral and central parts, with enhancement defects; (3) peripheral hyperenhancement: irregular rim-like hyperenhancement at the periphery of the lesion, with sparse filiform and punctiform internal enhancement; and (4) isoenhancement/hypoenhancement: enhancement of the lesion to a similar or lesser degree compared with the liver parenchyma.

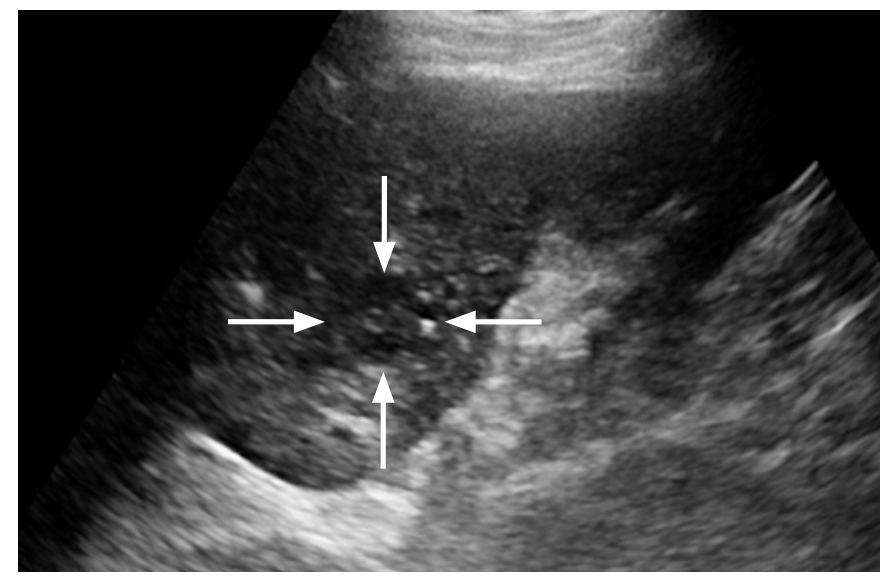

A

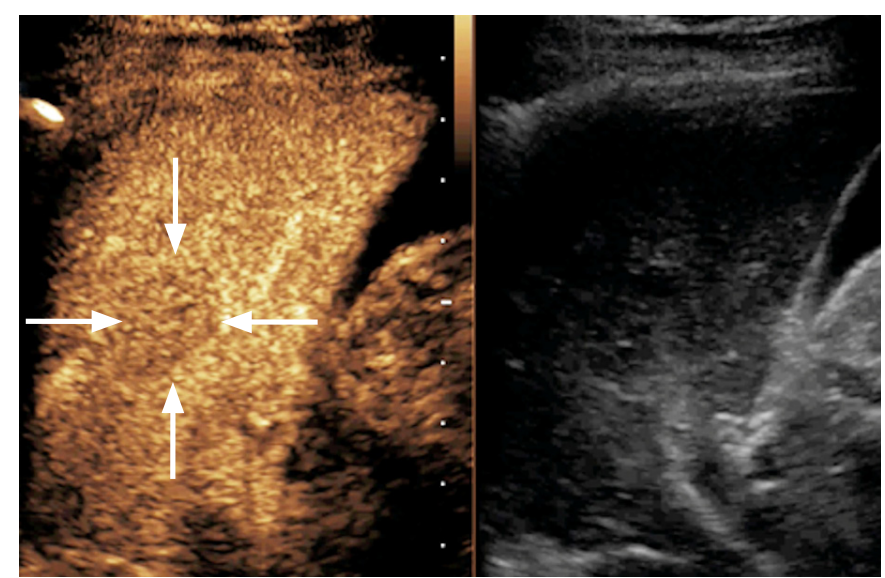

C
In the portal and delayed phases, the presence of washout and washout degree were evaluated. Washout was defined as hypoenhancement of the lesion in the portal or delayed phase preceded by arterial hyperenhancement. In patients with arterial peripheral or heterogeneous hyperenhancement, washout was confined to the hyperenhanced portion within the lesion. The washout degree in the portal and delayed phase was classified as marked washout (obviously lower echogenicity than that of the liver parenchyma), mild washout (slight hypoechogenicity compared to the surrounding liver parenchyma) and no washout (similar or slightly higher echogenicity relative to the liver parenchyma preceded by hyperenhancement in the arterial phase).

Furthermore, time-related CEUS parameters were visually recorded. Enhancement time (ET) was defined as the time interval between the contrast agent injection ( 0 second) and its emergence
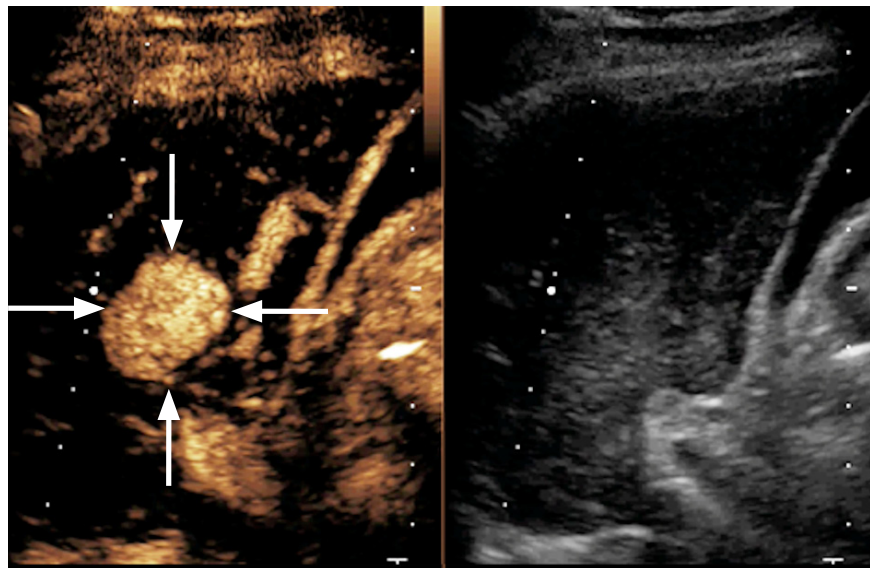

B

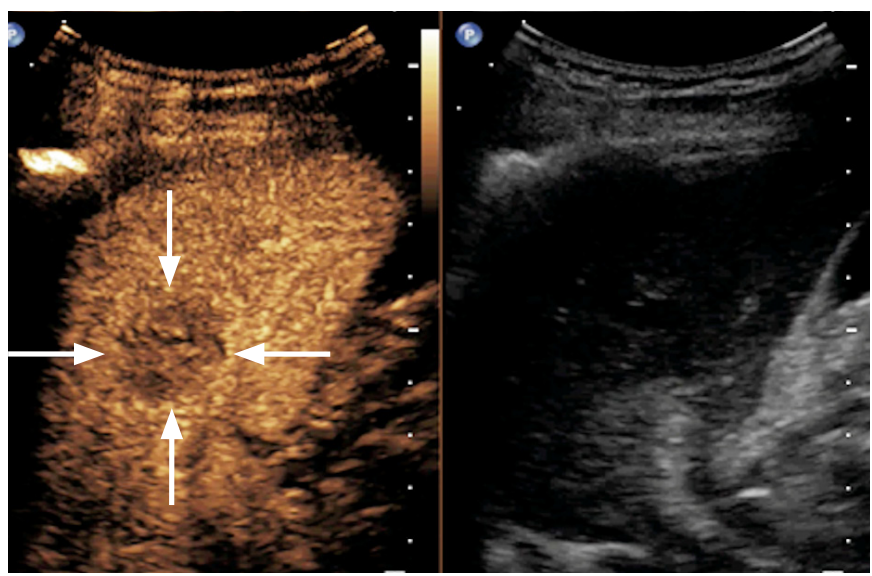

D

Fig. 1. Contrast-enhanced ultrasound imaging in a 51-year-old woman with pathologically proven hepatocellular carcinoma (arrows). A. On grayscale ultrasonography, a small hypoechoic lesion measuring $22 \mathrm{~mm}$ is demonstrated in the right lobe. B. In the arterial phase, the lesion shows homogeneous hyperenhancement (21 seconds). C, D. In the portal and delayed phase, the lesion shows mild washout (95 seconds at C, and 166 seconds at D). 


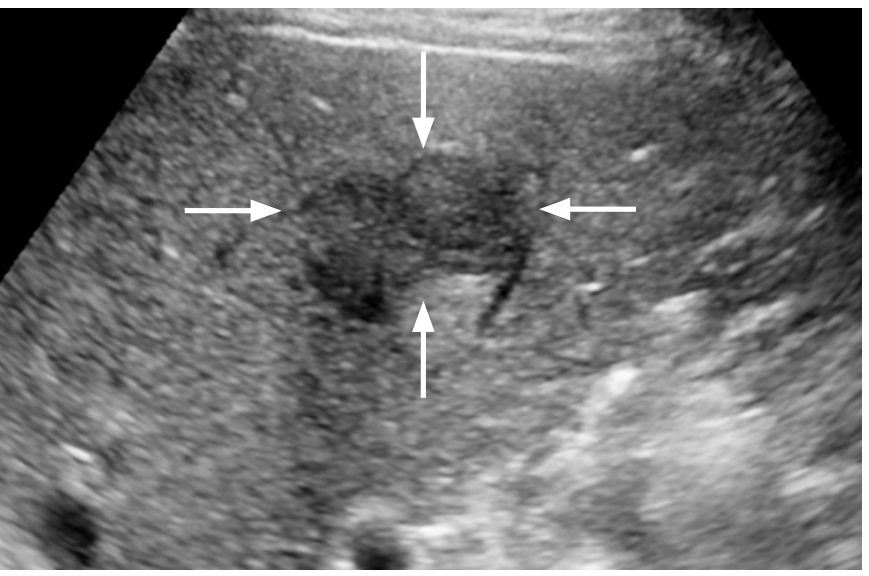

A

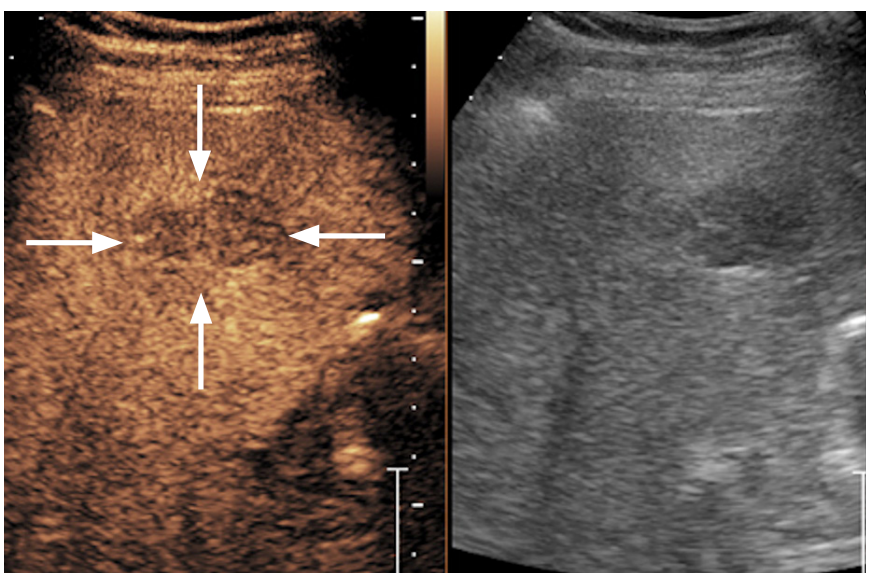

C

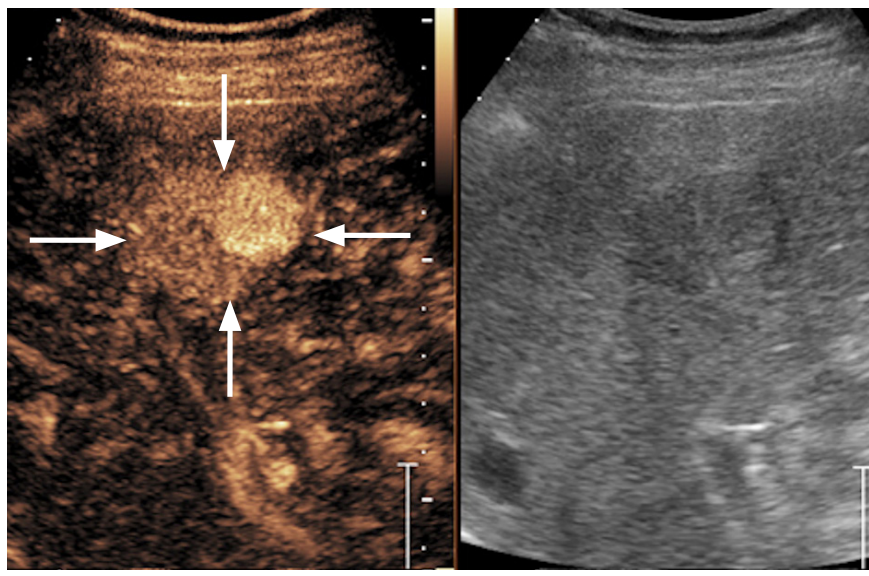

B

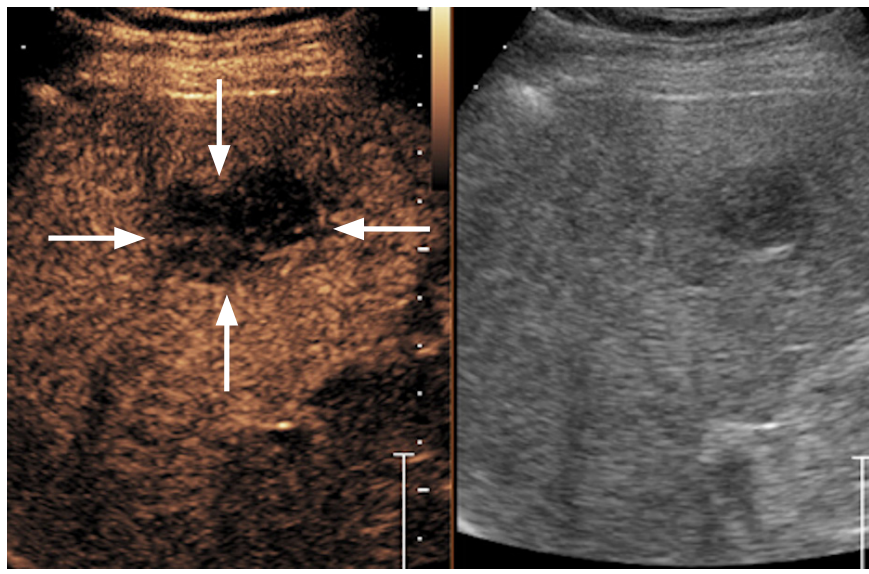

D

Fig. 2. Contrast-enhanced ultrasound (CEUS) imaging in a 56-year-old man with pathologically proven combined hepatocellular cholangiocarcinoma (arrows).

A. On grayscale ultrasonography, a lobulated hypoechoic lesion measuring $46 \times 35 \mathrm{~mm}$ is detected in the right lobe. B. A CEUS image reveals homogeneous hyperenhancement in the arterial phase ( 21 seconds). C. Mild washout is observed in the portal phase (40 seconds). D. In the delayed phase, the lesion demonstrates marked washout (150 seconds).

within the lesion; time to peak (TTP) was defined as the time interval between the emergence of contrast agent within the lesion and peak enhancement; and washout time (WT) was defined as the time interval between the emergence of contrast agent and the time point of hypoechogenicity within the lesion.

\section{Statistical Analysis}

All statistical analyses were performed using SPSS version 24.0 (IBM Corp., Armonk, NY, USA). The clinical and CEUS characteristics of the patients were expressed as mean \pm standard deviation and range or as count and proportion. The chi-square test or Fisher exact test was applied to compare differences in categorical variables. The independent-sample $t$ test was used to compare differences in timerelated parameters, including ET, TTP, and WT. P-values of $<0.05$ were considered to indicate statistically significant differences. We calculated the accuracy, sensitivity, and specificity of the features that played a statistically significant role in the differential diagnosis.

\section{Results}

\section{Clinical Characteristics}

The comparisons of the clinical characteristics of $\mathrm{CHC}, \mathrm{HCC}$, and $\mathrm{CC}$ are summarized in Table 1. The percentage of hepatitis B infections and the percentage of patients with a fibrotic or cirrhotic hepatic background showed no significant differences among the three entities. Elevated AFP was more common in CHC (55.6\%) and HCC (71.1\%) than in CC (2.2\%), while CA 19-9 was more common in CHC $(28.9 \%)$ and CC $(40.0 \%)$ than in HCC (2.2\%). Simultaneous elevation of AFP and CA 19-9 was observed in 17.8\% (8 of 45) of $\mathrm{CHCs}$ and $2.2 \%$ (1 of 45 ) of HCCs, and in no CCs. The tumor size of 


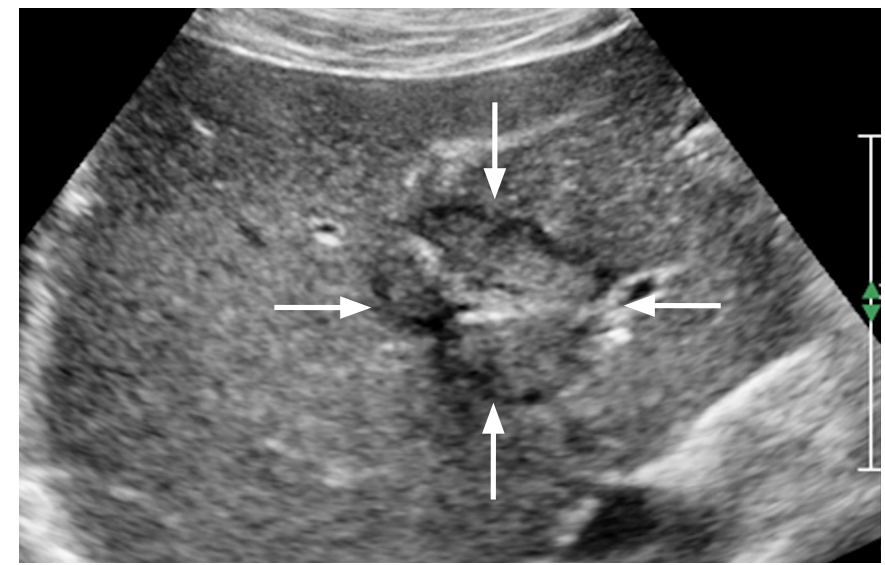

A

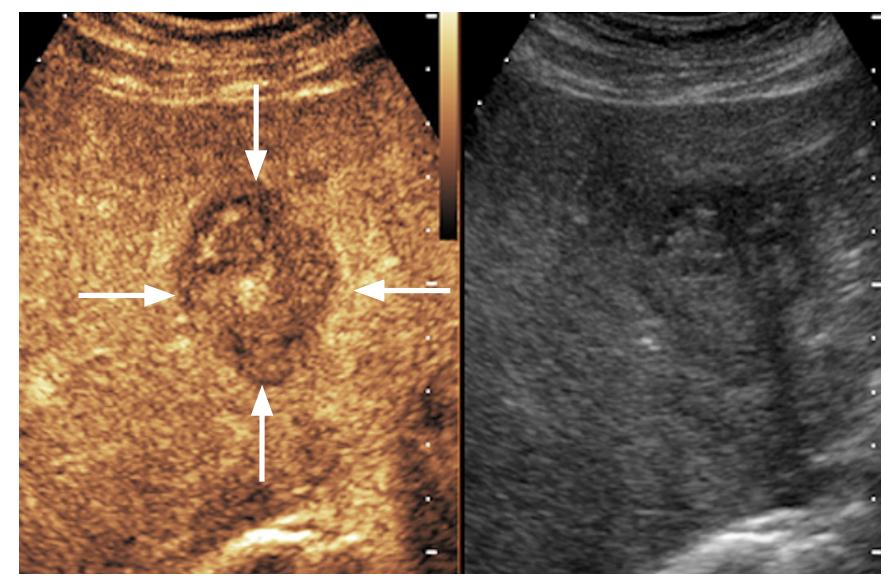

C

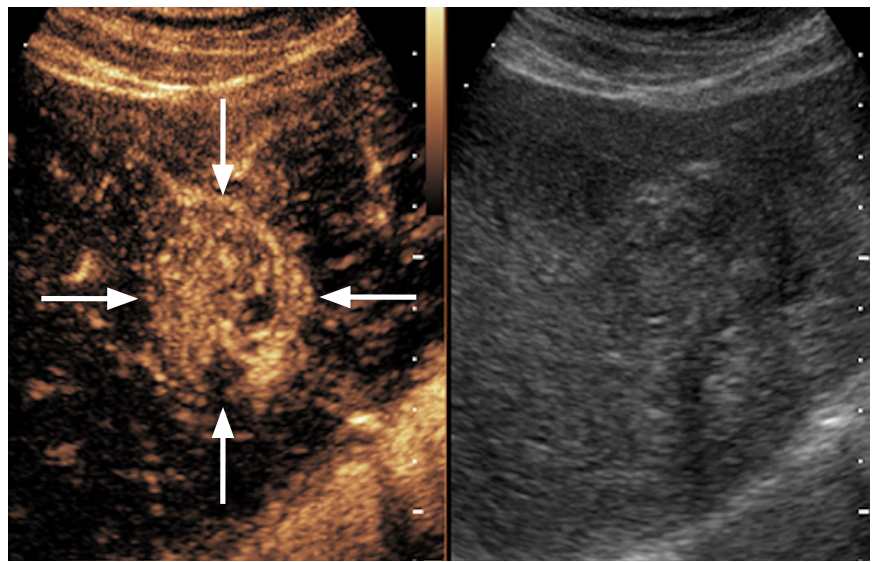

B

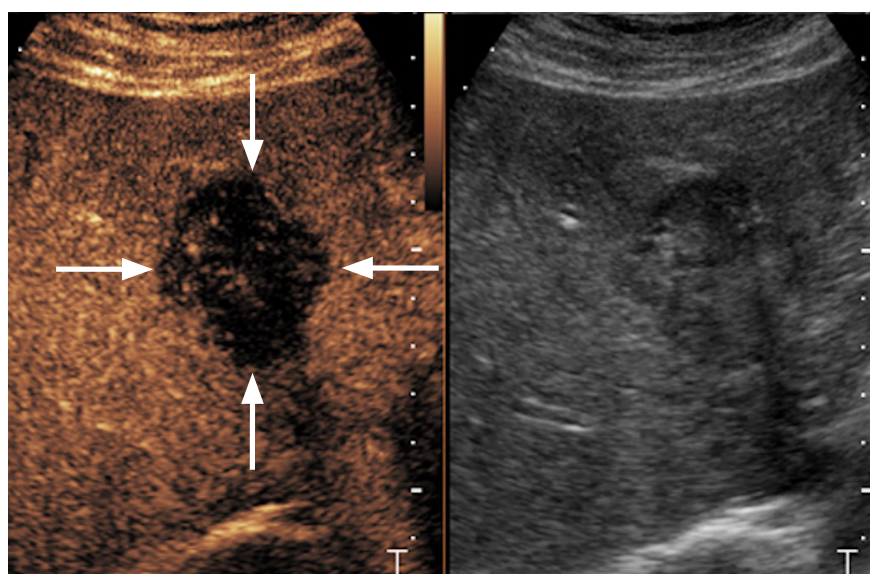

D

Fig. 3. Contrast-enhanced ultrasound (CEUS) imaging in a 51-year-old man with pathologically proven cholangiocarcinoma (arrows). A. On grayscale ultrasonography, the lesion demonstrates isoechogenicity with a lobulated shape and peripheral halo. The tumor size was $46 \times 38 \mathrm{~mm}$. B. A CEUS image reveals homogeneous hyperenhancement in the arterial phase (22 seconds). C. Mild washout is observed in the portal phase (38 seconds). D. In the delayed phase, the lesion demonstrates marked washout (142 seconds).

CHCs was comparable with that of HCCs $(P=0.247)$, while it was significantly smaller than that of CCs $(P=0.035)$.

\section{CEUS Imaging Features}

The CEUS imaging features of the three entities are summarized in Table 2.

In the arterial phase, hyperenhancement (either homogeneous or heterogeneous) was much more common in $\mathrm{CHC}$ and $\mathrm{HCC}$ than in CC $(73.3 \%$ and $100 \%$ vs. $37.8 \%)$, while peripheral enhancement was predominantly displayed in CC $(62.2 \%$ vs. $0 \%$ in HCC and $26.7 \%$ in $\mathrm{CHC})$.

In the portal phase, washout was detected in $95.6 \%$ of $\mathrm{CHCS}$, $62.2 \%$ of HCCs, and $100 \%$ of CCs. Additionally, the frequency of marked washout was much higher in $\mathrm{CHC}(42.2 \%)$ than in $\mathrm{HCC}$ $(6.7 \%)(\mathrm{P}<0.001)$, and was comparable to that in $\mathrm{CC}(53.3 \%)$
$(P=0.291)$.

In the delayed phase, the frequency of marked washout in CHC and CC was comparable (82.2\% vs. $93.3 \%, \mathrm{P}=0.108)$, and significantly higher than in HCC $(40.0 \%)$. The majority of HCCS displayed mild washout (57.8\%).

Therefore, the most common enhancement pattern of $\mathrm{CHC}$ was hyperenhancement (homogeneous or heterogeneous) in the arterial phase followed by marked washout in the delayed phase, and the second most common enhancement pattern of $\mathrm{CHC}$ was peripheral hyperenhancement in the arterial phase followed by marked washout in the delayed phase. However, based on the enhancement pattern, eight and $12 \mathrm{CHCs}$ were misdiagnosed as HCCS and CCs, respectively, because they showed a typical HCC enhancement pattern (hyperenhancement with mild washout in the delayed phase) or a typical CC enhancement pattern (peripheral 


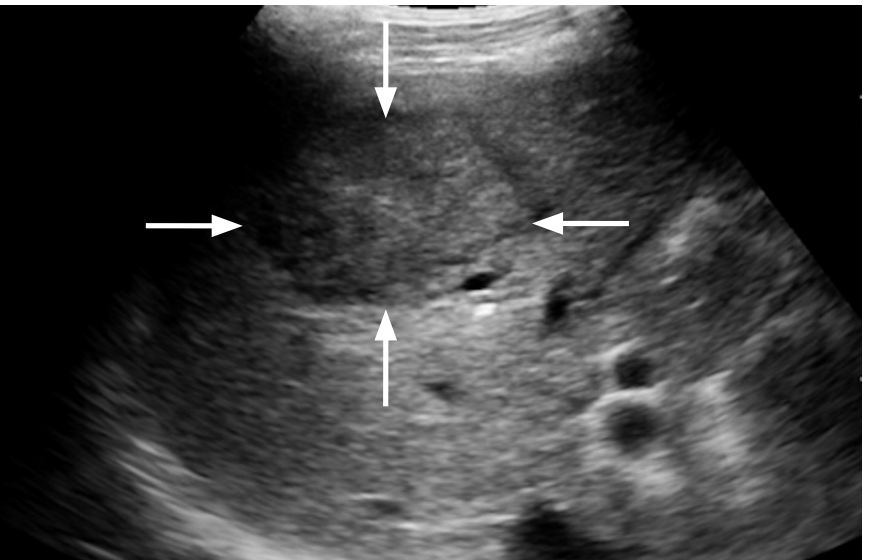

A

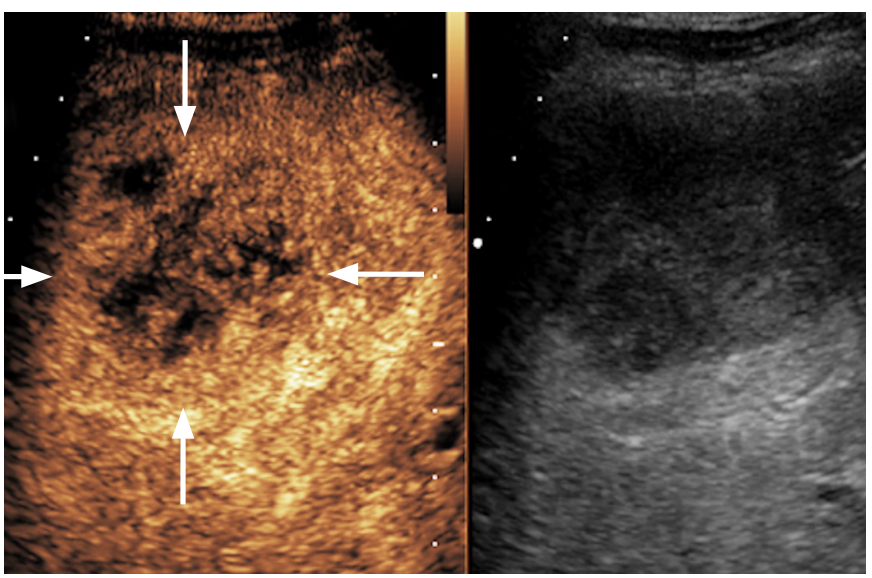

C

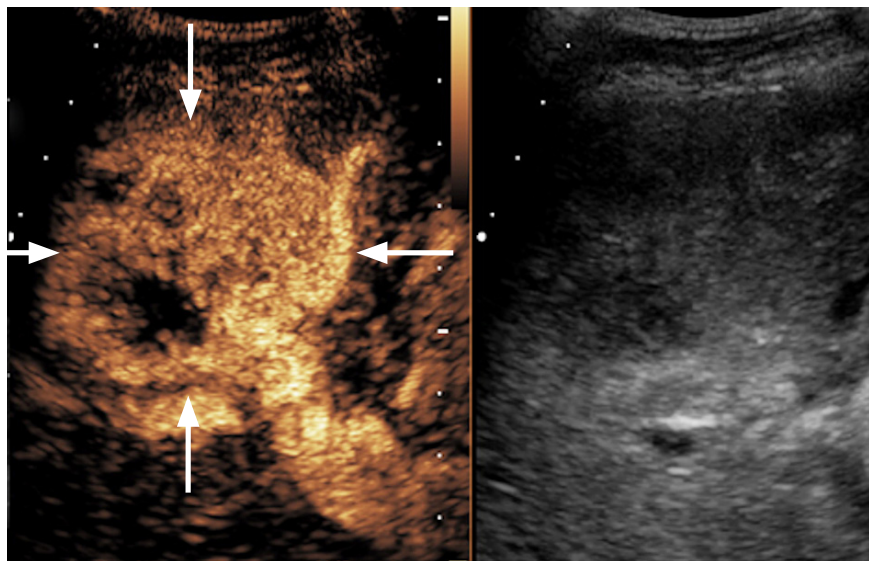

B

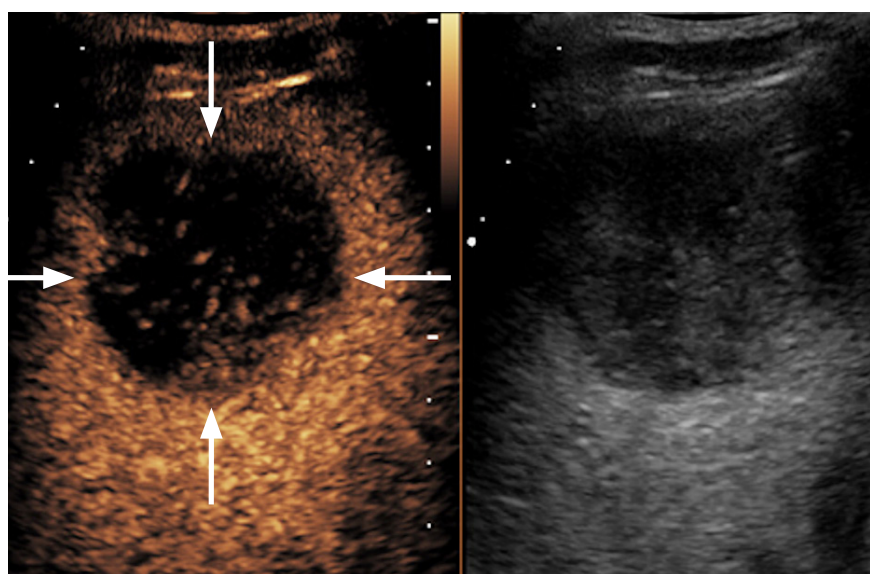

D

Fig. 4. Contrast-enhanced ultrasound (CEUS) imaging in a 62-year-old man with pathologically proven cholangiocarcinoma (arrows). A. Grayscale ultrasonography demonstrates a hypoechoic round lesion measuring $51 \times 49 \mathrm{~mm}$. B. In the arterial phase, a CEUS image reveals heterogeneous hyperenhancement ( 24 seconds). C. Rapid washout is observed in the portal phase (44 seconds). D. In the delayed phase, the lesion demonstrates marked washout (150 seconds).

enhancement in the arterial phase with marked washout in the delayed phase).

Of the time-related CEUS parameters, ET and TTP showed no significant differences among the three different entities. The WT in CHC (33.8 \pm 13.1 seconds) was comparable to that in CC $(30.1 \pm 11.6$ seconds) $(P=0.229)$, but was much shorter than that in HCC (58.4 \pm 23.5 seconds) $(\mathrm{P}=0.002)$.

\section{Diagnostic Efficacy of CEUS and Clinical Features}

The diagnostic efficacy of CEUS features and clinical tumor markers is presented in Table 3.

For differentiating between $\mathrm{CHC}$ and $\mathrm{HCC}$, the combination of the enhancement pattern, tumor marker, and WT showed the highest diagnostic value, with accuracy, sensitivity, and specificity of $74.4 \%$, $93.3 \%$, and $55.6 \%$, respectively.
For differentiating between $\mathrm{CHC}$ and $\mathrm{CC}$, this combination also showed higher efficacy than CEUS enhancement features and tumor markers alone, with accuracy, sensitivity, and specificity of $71.1 \%$, $80.0 \%$, and $62.2 \%$, respectively.

\section{Discussion}

With the wide application of ultrasound contrast agents in clinical practice, CEUS has notably improved the diagnostic performance of ultrasonography for several diseases, especially for the differentiation of focal liver lesions, with diagnostic performance that is even comparable to that of CECT and MRI [18]. Thus, in this retrospective study, the value of CEUS in combination with clinical features for differentiating between $\mathrm{CHC}, \mathrm{HCC}$, and CC was evaluated, thereby making a novel contribution to the literature. 


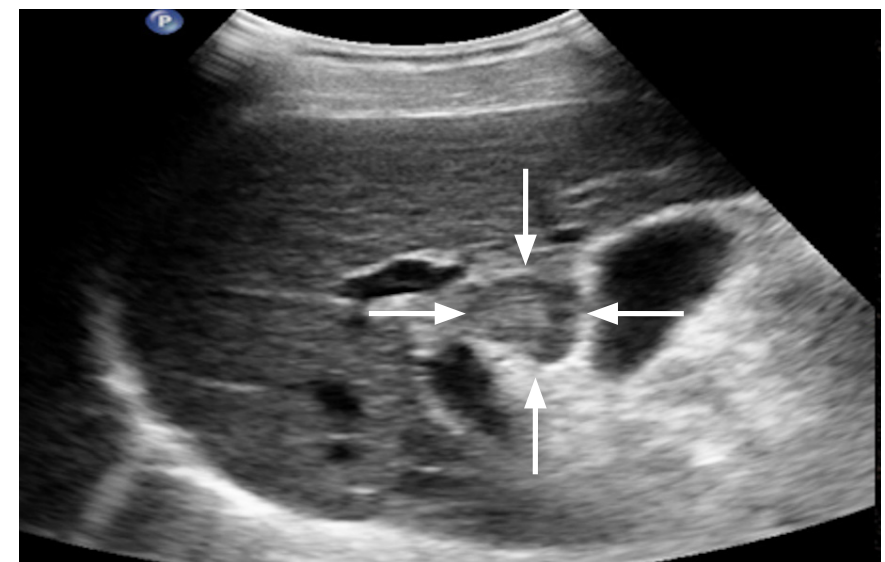

A

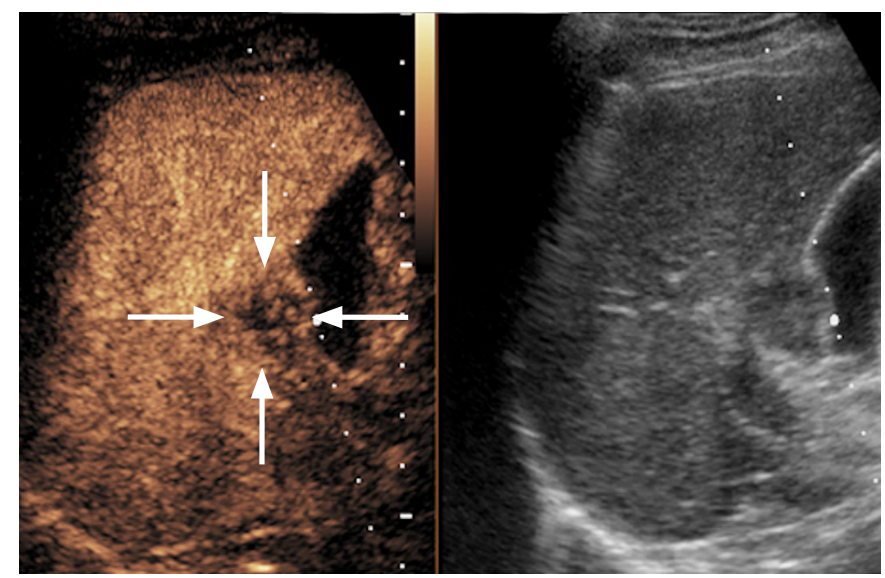

C

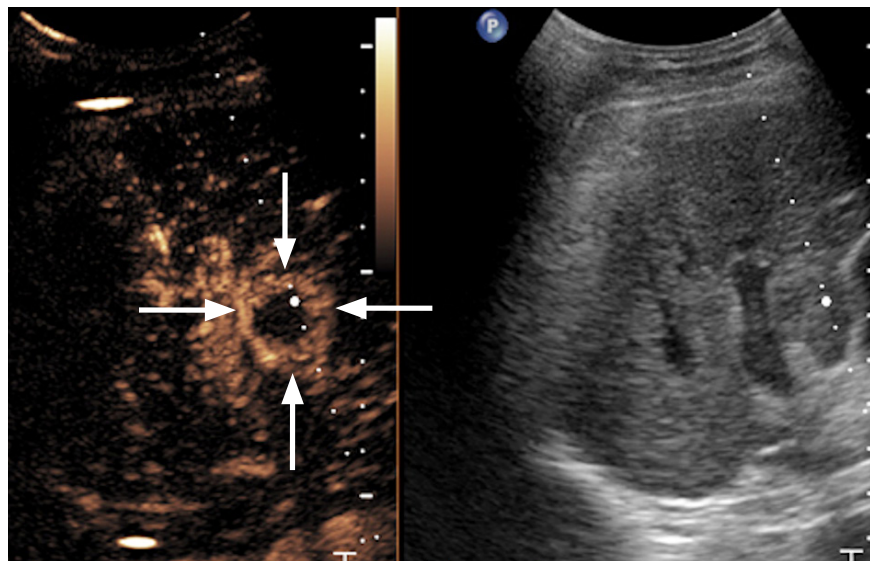

B

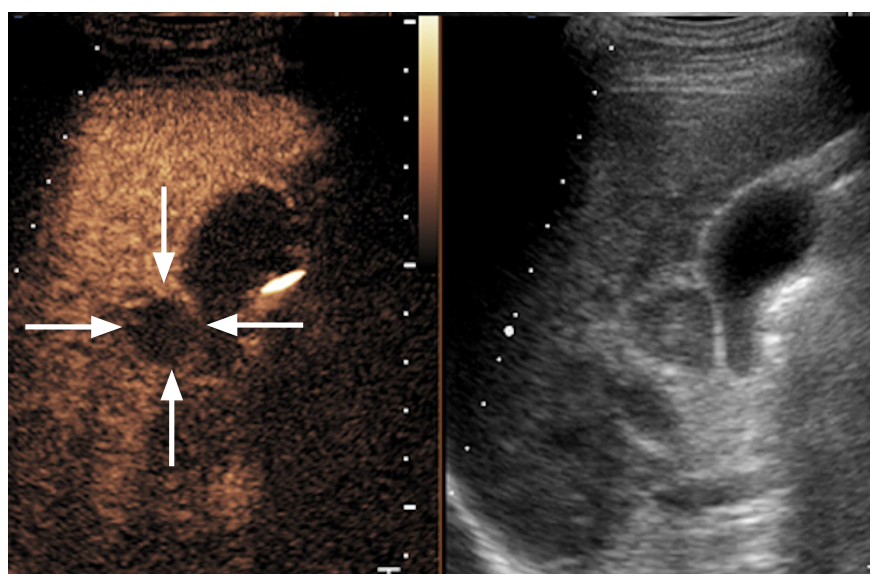

D

Fig. 5. Contrast-enhanced ultrasound (CEUS) imaging in a 44-year-old woman with pathologically proven combined hepatocellular cholangiocarcinoma (arrows).

A. On grayscale ultrasonography, a round hypoechoic lesion measuring $24 \times 20 \mathrm{~mm}$ is detected in the right lobe. B. A CEUS image reveals peripheral rim-like hyperenhancement in the arterial phase (18 seconds). C. Rapid washout is observed in the portal phase (47 seconds). D. In the delayed phase, the lesion demonstrates marked washout (132 seconds).

Regarding clinical features, although AFP and CA 19-9 are useful adjuncts for the diagnosis of HCC and CC, respectively [19], neither of them alone is sensitive or specific enough to identify CHC. However, the combination of these tumor markers may improve the sensitivity of the diagnosis. In present study, simultaneous elevation of AFP and CA 19-9 was more frequently detected in CHC (17.8\%) than that in $\mathrm{HCC}(2.2 \%)$ or CC $(0 \%)$, a pattern that is comparable with the findings of prior studies [20]. Although the sensitivity of this criterion was relatively low for differentiating these entities (17.8\%), the high specificity and negative predictive value demonstrated its strong ability to prevent $\mathrm{CHC}$ from being misdiagnosed as $\mathrm{HCC}$ or CC. In such cases, the diagnosis of $\mathrm{CHC}$ could be made with greater confidence.

In previous studies, the discordance between tumor marker elevation and imaging morphology (e.g., elevated CA 19-9 with imaging findings of $\mathrm{HCC}$, or elevated AFP with imaging findings of $\mathrm{CC}$ ) has been reported to be suggestive of $\mathrm{CHC}$ [8]. However, those results were based on the imaging features derived from CECT and dynamic contrast-enhanced MRI, in which the enhancement features of HCC and CC are clearly distinct, with arterial hyperenhancement followed by washout in the portal venous or equilibrium phase being characteristic of $\mathrm{HCC}[8,21]$, and peripheral arterial enhancement with progressive enhancement in the portal venous or equilibrium phase being characteristic of $\mathrm{CC}[8,22]$. However, considerable overlap of CEUS features between HCC and CC has been reported, in accordance with the present study. Thus, the discordance between CEUS features and tumor markers was not analyzed in our study.

Histologically, CHC is a combination of intermixed HCC and CC 
Table 1. Comparisons of the clinical characteristics of $\mathrm{CHC}, \mathrm{HCC}$, and CC

\begin{tabular}{|c|c|c|c|c|c|}
\hline Clinical feature & $\mathrm{CHC}$ & $\mathrm{HCC}$ & CC & P-value (CHC vs. HCC) & P-value (CHC vs. CC) \\
\hline Age (yr) & & & & 0.282 & 0.463 \\
\hline Mean $\pm S D$ & $52.2 \pm 10.8$ & $46.8 \pm 13.6$ & $54.7 \pm 15.2$ & & \\
\hline Range & $36-69$ & $28-76$ & $34-80$ & & \\
\hline Sex & & & & 0.362 & 0.180 \\
\hline Male/female & $33 / 12$ & 29/16 & $27 / 18$ & & \\
\hline Tumor size (cm) & & & & 0.247 & 0.035 \\
\hline Mean $\pm S D$ & $4.2 \pm 1.9$ & $3.7 \pm 2.4$ & $5.2 \pm 2.0$ & & \\
\hline Range & $1.9-6.5$ & $2.0-9.5$ & $3.3-8.0$ & & \\
\hline Tumor number & & & & 0.091 & 0.649 \\
\hline $1 / 2 />2$ & $30 / 3 / 12$ & $37 / 6 / 2$ & $32 / 4 / 9$ & & \\
\hline Tumor location ${ }^{\text {b) }}$ & & & & 0.479 & 0.206 \\
\hline Left/right/both & $5 / 39 / 1$ & $3 / 40 / 2$ & $9 / 33 / 3$ & & \\
\hline Hepatic background ${ }^{c)}$ & & & & 0.116 & 0.398 \\
\hline Cirrhosis & 26 & 31 & 22 & & \\
\hline Fibrosis & 15 & 11 & 16 & & \\
\hline Normal & 4 & 3 & 7 & & \\
\hline \multicolumn{6}{|l|}{ Tumor markers } \\
\hline Elevated AFP & 25 & 32 & 1 & 0.126 & $<0.001$ \\
\hline Elevated CA 19-9 & 13 & 1 & 18 & $<0.001$ & 0.267 \\
\hline Simultaneous elevation & 8 & 1 & 0 & 0.014 & 0.003 \\
\hline HBV infection & & & & 0.292 & 0.561 \\
\hline Positive & 39 & 42 & 37 & & \\
\hline Negative & 6 & 3 & 8 & & \\
\hline
\end{tabular}

CHC, combined hepatocellular cholangiocarcinoma; HCC, hepatocellular carcinoma; CC, cholangiocarcinoma; SD, standard deviation; AFP, a-fetoprotein; CA, cancer antigen; $\mathrm{HBV}$, hepatitis B virus.

${ }^{a)}$ Comparison between solitary and multiple lesions. ${ }^{b)}$ Comparison between left lobe and right lobe. ${ }^{\mathrm{c} C}$ Comparison between cirrhotic and non-cirrhotic background.

components. Therefore, the well-known imaging features of $\mathrm{HCC}$ and CC may provide a framework for approaching the diagnosis of $\mathrm{CHC}$ [8]. It has been reported that the ratio of HCC and CC components within the lesion can serve as a distinct CHC imaging appearance [8]. Arterial hyperenhancement followed by portal or delayed washout is considered to be the most characteristic CEUS feature of HCC $[5,23]$, and the common CEUS findings of CC include peripheral arterial rim-like enhancement with portal or delayed washout [23]. However, some CC lesions may also demonstrate similar enhancement patterns to those of HCC, especially in small lesions and those with a cirrhotic background [24], for which reason CEUS was dropped from the list of diagnostic techniques recommended for cirrhotic nodules in the guideline of the European Association for the Study of the Liver, European Organization for Research and Treatment of Cancer in 2012 [25]. Therefore, it is unreliable to use only the enhancement and washout pattern to differentiate $\mathrm{CHC}$ from HCC and $\mathrm{CC}$, since there are considerable overlaps between $\mathrm{CHC}$ and both CC and HCC.

In recent studies, the time and degree of washout have been proposed and proven important for differentiating between HCC and CC components $[14,15,20]$. In our study, although arterial hyperenhancement and the presence of washout in the portal and delayed phases showed no differences between HCC and CHC, a marked degree of washout in the delayed phase was much common in CHC than in HCC $(82.2 \%$ vs. $40.0 \%)$. Li et al. [14] reported that marked washout in the delayed phase was present in $76 \%$ of $\mathrm{CHCS}$, but only in $10 \%$ of HCCs. Therefore, marked washout in the delayed phase may have the potential to provide diagnostic clues for CHC. In our study, when marked washout in the delayed phase was used as a criterion for differentiating $\mathrm{CHC}$ from HCC, the sensitivity and specificity were $82.2 \%$ and $60.0 \%$, respectively. However, the corresponding values reported by Li et al. [14] were much higher (78\% and $90 \%$, respectively). This discrepancy may be due to differences in the tumor size, tumor differentiation, hepatic 
Table 2. Comparisons of the CEUS enhancement features of $\mathrm{CHC}$, HCC, and CC

\begin{tabular}{|c|c|c|c|c|c|}
\hline CEUS enhancement feature & $\mathrm{CHC}$ & $\mathrm{HCC}$ & CC & P-value (CHC vs. HCC) & P-value (CHC vs. CC) \\
\hline Arterial phase $e^{a)}$ & & & & $<0.001$ & 0.001 \\
\hline Homogeneous hyperenhancement & 21 & 29 & 8 & & \\
\hline Heterogeneous hyperenhancement & 12 & 16 & 9 & & \\
\hline Peripheral enhancement & 12 & 0 & 28 & & \\
\hline Isoenhancement/hypoenhancement & 0 & 0 & 0 & & \\
\hline Portal phase ${ }^{\text {b) }}$ & & & & $<0.001$ & 0.291 \\
\hline Mild washout & 24 & 25 & 21 & & \\
\hline Marked washout & 19 & 3 & 24 & & \\
\hline No washout & 2 & 17 & 0 & & \\
\hline Delayed phase ${ }^{c}$ & & & & $<0.001$ & 0.108 \\
\hline Mild washout & 8 & 26 & 3 & & \\
\hline Marked washout & 37 & 18 & 42 & & \\
\hline No washout & 0 & 1 & 0 & & \\
\hline Enhancement time (sec) & $20.3 \pm 3.6$ & $19.7 \pm 4.4$ & $19.0 \pm 3.9$ & 0.420 & 0.496 \\
\hline Time to peak (sec) & $13.9 \pm 4.4$ & $15.0 \pm 5.1$ & $13.2 \pm 4.2$ & 0.313 & 0.681 \\
\hline Washout time (sec) & $33.8 \pm 13.1$ & $58.4 \pm 23.5$ & $30.1 \pm 11.6$ & 0.002 & 0.229 \\
\hline
\end{tabular}

CEUS, contrast-enhanced ultrasonography; $\mathrm{CHC}$, combined hepatocellular cholangiocarcinoma; $\mathrm{HCC}$, hepatocellular carcinoma; CC, cholangiocarcinoma.

${ }^{a)}$ Comparison between peripheral enhancement and hyperenhancement (homogeneous and heterogeneous hyperenhancement). ${ }^{\text {b) }}$ Comparison between marked washout and no/mild washout. ${ }^{\mathrm{c}}$ Comparison between marked washout and no/mild washout.

Table 3. Diagnostic efficacy of CEUS and clinical features in the differential diagnosis

\begin{tabular}{|c|c|c|c|}
\hline & $\begin{array}{l}\text { Accuracy } \\
(\%)\end{array}$ & $\begin{array}{c}\text { Sensitivity } \\
\text { (\%) }\end{array}$ & $\begin{array}{l}\text { Specificity } \\
(\%)\end{array}$ \\
\hline \multicolumn{4}{|l|}{ CHC vs. HCC } \\
\hline $\begin{array}{l}\text { Marked washout in the delayed } \\
\text { phase }\end{array}$ & 71.1 & 82.2 & 60.0 \\
\hline $\begin{array}{l}\text { Simultaneous elevation of AFP and } \\
\text { CA 19-9 }\end{array}$ & 57.8 & 17.8 & 97.8 \\
\hline $\mathrm{WT}<38 \mathrm{sec}$ & 70.0 & 57.8 & 82.2 \\
\hline Combination & 74.4 & 93.3 & 55.6 \\
\hline \multicolumn{4}{|l|}{ CHC vs. CC } \\
\hline $\begin{array}{l}\text { Hyperenhancement in the arterial } \\
\text { phase }\end{array}$ & 67.8 & 73.3 & 62.2 \\
\hline $\begin{array}{l}\text { Simultaneous elevation of AFP and } \\
\text { CA 19-9 }\end{array}$ & 58.9 & 17.8 & 100 \\
\hline Combination & 71.1 & 80.0 & 62.2 \\
\hline
\end{tabular}

CEUS, contrast-enhanced ultrasonography; CHC, combined hepatocellular cholangiocarcinoma; HCC, hepatocellular carcinoma; AFP, a-fetoprotein; CA, cancer antigen; WT, washout time; CC, cholangiocarcinoma.

background, ratio of HCC and $\mathrm{CC}$ components within $\mathrm{CHC}$, and other factors between the two studies. The value of WT has not yet been explored in the diagnosis of CHC. According to previous studies on the differential diagnosis of HCC and CC, early washout ( $<60$ seconds) was more common in CC than in HCC $(87.9 \%$ vs.
$16.0 \%)$ [26], and the majority of CCs displayed washout within 43 seconds $[20,26,27]$. In our study, the WT was much shorter in CHC than in HCC ( $33.8 \pm 13.1$ seconds vs. $58.4 \pm 20.5$ seconds), and $57.8 \%$ (26 of 45 ) of CHCs displayed a WT $<38$ seconds, versus only $17.8 \%$ (8 of 45) of HCCs. However, in our study, WT was calculated differently from previous studies. Since the time of the emergence of contrast agent within the lesion may vary considerably due to individual differences in cardiac function, we defined WT as the interval between the emergence of contrast agent in the lesion and hypoenhancement, while in other studies, time 0 was set at the injection of the contrast agent. Nevertheless, the basic pattern of more rapid washout in $\mathrm{CHC}$ than in HCC remains clear. In our study, by using the combination of marked washout in the delayed phase, a WT $<38$ seconds, and simultaneous elevation of AFP and CA 19-9 to differentiate $\mathrm{CHC}$ from $\mathrm{HCC}$, the sensitivity and specificity could be increased to $93.3 \%$ and $55.6 \%$, respectively. However, since the ratio of histologically predominant components within the lesion may vary considerably, a larger sample size of CHCs should be studied in the future.

Concerning the CEUS features of CHC and CC, washout degree in the portal and delayed phases, as well as WT, showed no significant differences. In the arterial phase, hyperenhancement was more common in $\mathrm{CHC}$ than in CC $(73.3 \%$ vs. $37.8 \%, \mathrm{P}=0.001)$. Ye et al. [16] also demonstrated that peripheral rim-like arterial 
enhancement was an independent risk factor for $\mathrm{CHC}$. When arterial hyperenhancement was used as the criterion to differentiate $\mathrm{CHC}$ from CC, the sensitivity and specificity in our study were $73.3 \%$ and $62.2 \%$, respectively. The sensitivity was higher, and the specificity was somewhat lower than the values reported by Li et al. [14] (55\% and $78 \%$, respectively). However, in the study of Li et al. [14], the range of tumor size was large $(6 \mathrm{~cm})$, and the patient population was relatively small ( $30 \mathrm{CHCs}, 30 \mathrm{HCCs}$, and $32 \mathrm{CCs}$ ). Furthermore, the prevalence of cirrhosis in patients with $\mathrm{CHC}$ and $\mathrm{HCC}$ was much higher than that in patients with CC (52\% and $60 \%$ vs. $22 \%)$ in their study [14], while in our study, the prevalence of cirrhosis for each entity was comparable and was much higher than was observed for the corresponding types in the study of Li et al. [14]. This may cause differences in the diagnostic efficacy of CEUS feature of hyperenhancement in the differential diagnosis, because it has been reported that smaller tumors and higher frequencies of liver cirrhosis are associated with a higher likelihood of detecting arterial hyperenhancement within CC lesions [24]. This hypothesis should be further validated with studies concerning the CEUS features of CHCs with differences in size and hepatic background. Using the combination of simultaneous elevation of AFP and CA 19-9, the sensitivity and specificity were $80.0 \%$ and $62.2 \%$, respectively.

There are some limitations of our study that should be noted. First, the sample size of $\mathrm{CHC}$ was relatively small due to its rarity, and the influence of tumor size and hepatic background on CEUS features was not analyzed. Second, due to the nature of the retrospective study design, correlations between the CEUS features of CHC and histopathological findings were not investigated. Furthermore, we separately compared CEUS features between CHC and HCC and between $\mathrm{CHC}$ and $\mathrm{CC}$, and an intermingled differential diagnosis among the three tumor types was not conducted. Third, only CEUS was used to differentiate $\mathrm{CHC}$ from HCC and CC in our study, but more imaging modalities should be utilized to improve the diagnostic efficacy, such as CECT, dynamic contrast-enhanced MRI, and some other functional imaging modalities, including parametric imaging and positron emission tomography.

In conclusion, although the CEUS features of CHC, HCC, and CC may overlap, the combination of tumor markers, marked washout in the delayed phase, and a WT $<38$ seconds was confirmed to be helpful for differentiating between $\mathrm{CHC}$ and HCC, and the combination of tumor markers and arterial hyperenhancement may provide a differential diagnostic clue between $\mathrm{CHC}$ and CC.

ORCID: Hai-Chun Zhang: https://orcid.org/0000-0002-2457-5373; Ting Zhu: https:// orcid.org/0000-0001-8485-8095; Rong-Fei Hu: https://orcid.org/0000-0002-8800609X; Long Wu: https://orcid.org/0000-0002-4600-4977

\section{Author Contributions}

Conceptualization: Zhang HC. Data acquisition: Zhu T. Data analysis or interpretation: Wu L. Drafting of the manuscript: Zhang HC. Critical revision of the manuscript: Hu RF. Approval of the final version of the manuscript: all authors.

\section{Conflict of Interest}

No potential conflict of interest relevant to this article was reported.

\section{References}

1. Allen RA, Lisa JR. Combined liver cell and bile duct carcinoma. Am J Pathol 1949;25:647-655.

2. Jarnagin WR, Weber S, Tickoo SK, Koea JB, Obiekwe S, Fong Y, et al. Combined hepatocellular and cholangiocarcinoma: demographic, clinical, and prognostic factors. Cancer 2002;94:2040-2046.

3. Lee JH, Chung GE, Yu SJ, Hwang SY, Kim JS, Kim HY, et al. Longterm prognosis of combined hepatocellular and cholangiocarcinoma after curative resection comparison with hepatocellular carcinoma and cholangiocarcinoma. J Clin Gastroenterol 2011;45:69-75.

4. Chi M, Mikhitarian K, Shi C, Goff LW. Management of combined hepatocellular-cholangiocarcinoma: a case report and literature review. Gastrointest Cancer Res 2012;5:199-202.

5. Bruix J, Sherman M; American Association for the Study of Liver Diseases. Management of hepatocellular carcinoma: an update. Hepatology 2011;53:1020-1022.

6. Bridgewater J, Galle PR, Khan SA, Llovet JM, Park JW, Patel T, et al. Guidelines for the diagnosis and management of intrahepatic cholangiocarcinoma. J Hepatol 2014;60:1268-1289.

7. Yin X, Zhang BH, Qiu SJ, Ren ZG, Zhou J, Chen XH, et al. Combined hepatocellular carcinoma and cholangiocarcinoma: clinical features, treatment modalities, and prognosis. Ann Surg Oncol 2012;19:2869-2876.

8. Fowler KJ, Sheybani A, Parker RA 3rd, Doherty S, Brunt EM, Chapman WC, et al. Combined hepatocellular and cholangiocarcinoma (biphenotypic) tumors: imaging features and diagnostic accuracy of contrast-enhanced CT and MRI. AJR Am J Roentgenol 2013;201:332-339.

9. Ebied O, Federle MP, Blachar A, Brancatelli G, Grazioli L, CazalsHatem D, et al. Hepatocellular-cholangiocarcinoma: helical computed tomography findings in 30 patients. J Comput Assist Tomogr 2003;27:117-124.

10. Sheng RF, Xie $Y H$, Ji $Y$, Chen $C Z$, Yang $L$, Jin $K P$, et al. $M R$ comparative study of combined hepatocellular-cholangiocarcinoma in normal, fibrotic, and cirrhotic livers. Abdom Radiol (NY) 2016;41:2102-2114.

11. Park SH, Lee SS, Yu E, Kang HJ, Park Y, Kim SY, et al. Combined hepatocellular-cholangiocarcinoma: gadoxetic acid-enhanced MRI 
findings correlated with pathologic features and prognosis. J Magn Reson Imaging 2017;46:267-280.

12. Ferraioli G, Meloni MF. Contrast-enhanced ultrasonography of the liver using SonoVue. Ultrasonography 2018;37:25-35.

13. Bartolotta TV, Taibbi A, Midiri M, Lagalla R. Contrast-enhanced ultrasound of hepatocellular carcinoma: where do we stand? Ultrasonography 2019;38:200-214.

14. Li F, Han J, Han F, Wang JW, Luo RZ, Li AH, et al. Combined hepatocellular cholangiocarcinoma (biphenotypic) tumors: potential role of contrast-enhanced ultrasound in diagnosis. AJR Am J Roentgenol 2017;209:767-774.

15. Yang HK, Burns PN, Jang HJ, Kono Y, Khalili K, Wilson SR, et al. Contrast-enhanced ultrasound approach to the diagnosis of focal liver lesions: the importance of washout. Ultrasonography 2019;38:289-301.

16. Ye J, Xie $X$, Lin $Y$, Liu B, Wang W, Huang $X$, et al. Imaging features of combined hepatocellular-cholangiocarcinoma on contrastenhanced ultrasound: correlation with clinicopathological findings. Clin Radiol 2018;73:237-243.

17. de Campos RO, Semelka RC, Azevedo RM, Ramalho M, Heredia $V$, Armao DM, et al. Combined hepatocellular carcinomacholangiocarcinoma: report of MR appearance in eleven patients. J Magn Reson Imaging 2012;36:1139-1147.

18. Claudon M, Cosgrove D, Albrecht T, Bolondi L, Bosio M, Calliada F, et al. Guidelines and good clinical practice recommendations for contrast enhanced ultrasound (CEUS): update 2008. Ultraschall Med 2008;29:28-44.

19. Shetty AS, Fowler KJ, Brunt EM, Agarwal S, Narra VR, Menias CO. Combined hepatocellular-cholangiocarcinoma: what the radiologist needs to know about biphenotypic liver carcinoma. Abdom Imaging 2014;39:310-322.

20. Li R, Yang D, Tang CL, Cai P, Ma KS, Ding SY, et al. Combined hepatocellular carcinoma and cholangiocarcinoma (biphenotypic) tumors: clinical characteristics, imaging features of contrastenhanced ultrasound and computed tomography. BMC Cancer 2016;16:158.

21. Fowler KJ, Brown JJ, Narra VR. Magnetic resonance imaging of focal liver lesions: approach to imaging diagnosis. Hepatology 2011;54:2227-2237.

22. Nishie A, Yoshimitsu $K$, Asayama $Y$, Irie $H$, Aibe $H$, Tajima $T$, et al. Detection of combined hepatocellular and cholangiocarcinomas on enhanced CT: comparison with histologic findings. AJR Am J Roentgenol 2005;184:1157-1162.

23. Claudon M, Dietrich CF, Choi BI, Cosgrove DO, Kudo M, Nolsoe CP, et al. Guidelines and good clinical practice recommendations for contrast enhanced ultrasound (CEUS) in the liver--update 2012: a WFUMB-EFSUMB initiative in cooperation with representatives of AFSUMB, AIUM, ASUM, FLAUS and ICUS. Ultraschall Med 2013;34:11-29.

24. Lu Q, Xue LY, Wang WP, Huang BJ, Li CX. Dynamic enhancement pattern of intrahepatic cholangiocarcinoma on contrast-enhanced ultrasound: the correlation with cirrhosis and tumor size. Abdom Imaging 2015;40:1558-1566.

25. European Association for the Study of the Liver; European Organisation for Research and Treatment of Cancer. EASLEORTC clinical practice guidelines: management of hepatocellular carcinoma. J Hepatol 2012;56:908-943.

26. Li R, Yuan MX, Ma KS, Li XW, Tang CL, Zhang XH, et al. Detailed analysis of temporal features on contrast enhanced ultrasound may help differentiate intrahepatic cholangiocarcinoma from hepatocellular carcinoma in cirrhosis. PLoS One 2014;9:e98612.

27. Liu GJ, Wang W, Lu MD, Xie XY, Xu HX, Xu ZF, et al. Contrastenhanced ultrasound for the characterization of hepatocellular carcinoma and intrahepatic cholangiocarcinoma. Liver Cancer 2015;4:241-252. 